\title{
BMJ Open Transitional care quality indicators to assess quality of care following hospitalisation for chronic obstructive pulmonary disease and heart failure: a systematic review protocol
}

\author{
Faith Michael (D) , ${ }^{1}$ Sera Whitelaw, ${ }^{2}$ Harriette GC Van Spall ${ }^{2,3,4}$
}

To cite: Michael F, Whitelaw S, Van Spall HGC. Transitional care quality indicators to assess quality of care following hospitalisation for chronic obstructive pulmonary disease and heart failure: a systematic review protocol. BMJ Open 2019;9:e032764. doi:10.1136/ bmjopen-2019-032764

- Prepublication history and additional material for this paper are available online. To view these files, please visit the journal online (http://dx.doi. org/10.1136/bmjopen-2019032764).

Received 04 July 2019 Revised 23 November 2019 Accepted 26 November 2019

\section{Check for updates}

(c) Author(s) (or their employer(s)) 2019. Re-use permitted under CC BY-NC. No commercial re-use. See rights and permissions. Published by BMJ.

${ }^{1}$ Michael G. DeGroote School of Medicine, McMaster University, Hamilton, Ontario, Canada

${ }^{2}$ Department of Health Research Methods, Evidence, and Impact, McMaster University, Hamilton, Ontario, Canada

${ }^{3}$ Population Health Research Institute, Hamilton, Ontario, Canada

${ }^{4}$ Department of Medicine, McMaster University, Hamilton, Ontario, Canada

Correspondence to Dr Harriette GC Van Spall; Harriette.VanSpall@phri.ca

\section{ABSTRACT}

Introduction The period following hospitalisation for chronic obstructive pulmonary disease (COPD) or heart failure (HF)—when patients transition between settings and clinicians - is one of high risk. Transitional care services that bridge the gap from hospital to home can improve outcomes, but there are no widely accepted indicators to assess their quality.

Methods and analysis In this systematic review, we will summarise transitional care quality indicators, and describe their associations with clinical, patientreported and cost outcomes. We will search MEDLINE, Embase, CINAHL and HealthSTAR, as well as grey literature and reference lists of included articles. We will screen all studies published between January 1990 and October 2019 that test an intervention that aims to improve the hospital-to-home transition for patients with COPD and/or HF; and measure at least one process (eg, medication errors), clinical (eg, hospital readmissions) or patient-reported (eg, health-related quality of life) outcome which will serve as a transitional care quality indicator . We will include randomised controlled trials, cohort studies, cross-sectional studies, interrupted time series studies and before-after studies. We will extract data in duplicate and classify transitional care quality indicators as structural, process-related or outcomerelated. When possible, we will assess associations between transitional care quality indicators and clinical outcomes. In anticipation of conceptual and statistical heterogeneity, we will provide a qualitative synthesis and narrative review of the results.

Ethics and dissemination This review will provide a list of transitional care quality indicators and their associations with clinical outcomes. These results can be used by hospitals, administrators and clinicians for assessing the quality of transitional care provided to patients with COPD and HF. The findings can also be used by policy-makers to assess and incentivise transitional care quality. We will disseminate results through publications, social media releases and presentations.

PROSPERO registration number This study is registered on PROSPERO.
Strengths and limitations of this study

- This study will generate a list of transitional care quality indicators that assess the quality of care as patients transition from hospital to home.

- The strength of this study includes its focus on the medical conditions that account for a majority of readmissions in older adults.

- The search strategy will be systematic, broad and comprehensive.

- The quality and availability of data in the primary research studies will limit the overall findings of this systematic review.

- The expected heterogeneity will likely preclude a meta-analysis and limit this study to a narrative review.

\section{INTRODUCTION}

Chronic obstructive pulmonary disease (COPD) and heart failure (HF) are the most common causes of hospitalisation among older adults ${ }^{1}$ and account for a large proportion of healthcare system costs in high-income countries. ${ }^{2} 3$ The period after hospital discharge is one of great vulnerability as patients transition between healthcare settings and clinicians, and learn to cope with their disease. Approximately one in five patients hospitalised for COPD or HF are readmitted within 30 days of discharge. ${ }^{4}$ Unplanned hospital readmissions burden patients, their caregivers and the healthcare system.

Organisations such as the National Quality Forum generate healthcare quality indicators with the goal of improving outcomes in conditions such as COPD and HF. ${ }^{5}$ Their quality indicator development process relies on individuals to submit indicators, and on stakeholders to approve the indicators via consensus. ${ }^{5}$ While this process engages 
stakeholders, it is effort-intensive. Furthermore, the association between the quality indicators and clinically meaningful outcomes is unclear; the evidence to support many transitional care quality indicators is uncertain, and using the wrong indicators can introduce measurement burden with no tangible improvement in patientimportant outcomes.

Transitional care services-those that provide coordination and continuity as patients transition between healthcare settings-can improve clinical outcomes following hospitalisation for COPD and HF. ${ }^{16}$ Healthcare authorities have prioritised efforts that improve the quality of transitional care in COPD and $\mathrm{HF}^{7}$ However, to date, there are no standard indicators to assess the quality of transitional care as patients transition from hospital to home. Such transitional care quality indicators, once identified, could be used to improve the quality of care provided to patients during this period. While several task forces have demonstrated interest in linking transitional care quality indicators with all-cause readmissions, ${ }^{8}$ this outcome alone can be misleading as it does not account for other important outcomes, such as emergency department (ED) visits or death.

In this systematic review, we will focus on transitional care quality indicators that assess care quality as patients transition from hospital to home. We will generate a comprehensive list of quality indicators and assess their associations with clinical outcomes. Transitional care quality indicators that have associations with meaningful outcomes can be used to measure the effectiveness of transitional care interventions in both research and clinical settings.

\section{METHODS AND ANALYSIS \\ Information sources}

We will conduct a systematic search of the literature, restricted to the English language, for articles that have been published between January 1990 and October 2019 in MEDLINE, Embase, CINAHL and HealthSTAR. We will search for additional studies that meet the specified eligibility criteria in grey literature databases. We will hand search the reference lists of relevant systematic reviews obtained from the literature searches and articles that meet the study's eligibility criteria.

\section{Search strategy}

Our search strategy will include the terms " 'chronic obstructive pulmonary disease', 'heart failure, 'chronic disease', 'health services' and 'hospitalization'. The preliminary search strategy for Embase is available in online supplementary appendix 1 . We will adapt and apply the Embase search strategy in the other databases of interest and, where applicable, use database-specific vocabulary.

\section{Eligibility criteria}

Inclusion criteria will follow the Population, Intervention, Comparison, Outcomes, Study framework. To generate a list of transitional care quality indicators, we will include studies with adult patients ( $\geq 19$ years old) hospitalised for COPD or HF. We will screen all studies that (1) test an intervention that aims to improve the hospital-to-home transition and (2) measure at least one of the following outcomes of interest: hospital readmissions, ED visits, mortality, length of subsequent hospitalisations, adverse medication events, healthcare costs and patient-reported outcomes. Both the intervention and the outcomes will be used to generate a list of transitional care quality indicators. For the process and patient-reported transitional care quality indicators, we will identify additional studies that describe the validation of the measurement tools to assess for associations with clinical outcomes, such as readmission or death. We will include randomised controlled trials (RCTs), cohort studies, cross-sectional studies, interrupted time series studies and before-after studies. We will exclude systematic reviews and meta-analyses (primary articles will be reviewed for inclusion instead), editorials, letters to the editor and studies published prior to 1990 .

\section{Study selection and data extraction}

We will use a reference manager software to identify and remove duplicate results retrieved from our literature searches. Two authors will independently screen the titles and abstracts of the studies to determine eligibility for inclusion in the review. If the information required to determine eligibility is not described in sufficient detail, we will attempt to contact the corresponding author for additional information. Disagreements between authors will be resolved through discussion, and when required, by consulting a third author. Two authors will independently evaluate the full texts of articles selected during preliminary screening and record reasons for exclusion of ineligible studies. The $\kappa$ statistic will be calculated to measure inter-rater agreement for a pilot screening of a random sample of 50 articles. If necessary, the inclusion and exclusion criteria will be clarified to promote consistent application of the eligibility criteria.

\section{Data extraction and management}

We will independently extract the following information in duplicate: study design, study setting, intervention, unit of intervention allocation (eg, patients, clinics), number of units in allocation, outcome measures, type of analysis and type of transitional care quality indicator (structure, process, outcome).${ }^{9}{ }^{10}$ We will extract data on the following outcomes: hospital readmissions, ED visits, mortality, length of subsequent hospitalisations, adverse medication events, healthcare costs and patient-reported outcomes. If a study reports unclear or incomplete data, we will attempt to contact the corresponding author to obtain the data. Any disagreements in data extraction will be resolved by discussion and consultation with a third author when necessary. 


\section{Assessment of risk of bias in included studies}

Two authors will independently assess the risk of bias of all included studies. For studies that report clinical outcomes, the criteria for assessing risk of bias will vary by study design. The Cochrane Effective Practice and Organisation of Care Review Group criteria will be used for RCTs, cluster RCTs, controlled before-after studies and interrupted time series. ${ }^{11}$ The National Institutes of Health tool will be used to assess the risk of bias in cohort, uncontrolled before-after and cross-sectional studies. ${ }^{12}$ We will summarise the risk of bias within studies using the tools appropriate to the study design, and across studies using Cochrane's recommendation for summary assessments. ${ }^{11}$ All disagreements will be resolved by discussion and, when required, by consulting a third author.

For studies that report patient-reported outcomes, the risk of bias assessment will focus on the validation of the tool. Studies that validate the tool will be obtained from the included studies, or from a search of the literature.

\section{Data synthesis}

We will use both interventions (eg, medication reconciliation at discharge) and outcomes (eg, discharge preparedness, as measured by the B-PREPARED score) to generate a list of transitional care quality indicators. We will classify each quality indicator as structure (assess physical care setting, including material and human resources), process (assess actions of providers) or outcome (assess impact of intervention on patient/population health)..$^{10}$ We will group studies by quality indicator and pool the results (associations between indicator and clinical, patient-reported or cost outcomes) for each transitional care quality indicator in a meta-analysis if the studies are sufficiently similar for pooling to be meaningful. Clinical heterogeneity will be assessed on the basis of included populations, intervention types and outcome measures. Statistical heterogeneity will be assessed with the $\mathrm{I}^{2}$ statistic. Substantial heterogeneity will be defined as $\mathrm{I}^{2}>75 \% .{ }^{13}$ In the absence of substantial heterogeneity, Cochrane Collaboration's Review Manager (RevMan) V.5.3.4 will be used to complete the meta-analyses. The pooled data will be computed using the Mantel-Haenszel method, under a random-effect model, and the 95\% confidence interval will be estimated. Forest plots will be used to display the results. However, in the likely circumstance that substantial heterogeneity prohibits the appropriate use of a meta-analysis, we will provide a qualitative synthesis of the transitional care quality indicators and outcomes in table format, accompanied by a narrative synthesis. We will analyse data according to study methodology and separate RCTs and observational studies, given their different levels of evidence.

\section{Patient and public involvement}

There are no patients involved in the design or conduct of this study.

\section{DISCUSSION AND DISSEMINATION}

This systematic review of adults hospitalised for COPD or HF will generate a list of transitional care quality indicators and summarise their associations with clinical outcomes. The study aim is relevant and pertains to the care of patients with diagnoses that account for a large proportion of care transitions in the healthcare system. The systematic design will allow for reliable conclusions to be drawn. The broad search strategy will enable the generation of a comprehensive list of transitional care quality indicators. The risk of bias assessment will help readers evaluate the quality of the evidence.

The results will be pertinent to numerous stakeholders. Hospitals can use the transitional care quality indicators to assess performance in preparing patients to return home after discharge. Researchers can use the findings to select outcomes for studies that evaluate the effect of transitional care interventions. The findings can prompt healthcare providers to adapt their practice, and can be used by health policy-makers to inform programs that incentivise the delivery of high-quality transitional care to improve patient and system outcomes.

This systematic review is not without limitations. We expect that the major limitation will be the design of the primary studies. To address this, we will assess the quality of each study. The search strategy is restricted to studies published in English. However, we will hand search included studies, relevant systematic reviews and grey literature databases to ensure literature saturation. The expected heterogeneity will likely preclude a metaanalysis and limit the conclusions that can be drawn. Another challenge will be achieving consensus on a core set of transitional care quality indicators. This will require the combined effort of healthcare providers, patients and stakeholders.

We will disseminate our results through publications in peer-reviewed journals, conference presentations and social media releases to encourage knowledge uptake at the provider, hospital and government levels.

Acknowledgments The authors thank Deepti Shanbhag and Maryan Graiss for their work with the search strategy.

Contributors HGCV obtained funding, conceived the research question and study design, informed the search strategy and analytic plan, and drafted and edited the manuscript. FM contributed to the search strategy, extracted data, and drafted and edited the manuscript. SW extracted data, and drafted and edited the manuscript. All authors read and approved the final manuscript.

Funding This research is funded by a grant from the Ontario's Ministry of Health and LongTerm Care (MOHLTC). HGCV receives support from the MOHLTC's Health System Research Fund and Hamilton Health Sciences Early Career Award.

\section{Competing interests None declared.}

Patient consent for publication Not required.

Provenance and peer review Not commissioned; externally peer reviewed.

Open access This is an open access article distributed in accordance with the Creative Commons Attribution Non Commercial (CC BY-NC 4.0) license, which permits others to distribute, remix, adapt, build upon this work non-commercially, and license their derivative works on different terms, provided the original work is properly cited, appropriate credit is given, any changes made indicated, and the use is non-commercial. See: http://creativecommons.org/licenses/by-nc/4.0/. 
ORCID iD

Faith Michael http://orcid.org/0000-0002-2331-4999

\section{REFERENCES}

1 Adeloye D, Chua S, Lee C, et al. Global and regional estimates of COPD prevalence: systematic review and meta-analysis. J Glob Health 2015;5.

2 Canadian Institute for Health Information. Inpatient hospitalizations, surgeries, newborns and childbirth indicators, 2016-2017. CIHI, 2018. Available: https://secure.cihi.ca/estore/productFamily.htm? locale=en\&pf=PFC3714\&lang=en [Accessed 26 Sep 2018].

3 Cook C, Cole G, Asaria P, et al. The annual global economic burden of heart failure. Int J Cardiol 2014;171:368-76.

4 Canadian Institute for Health Information. All-Cause readmission to acute care and return to the emergency department. Ottawa: $\mathrm{CIHI}$, 2012. Available: https://secure.cihi.ca/free_products/Readmission_ to_acutecare_en.pdf [Accessed 26 Sep 2018].

5 National Quality Forum. Measure applications partnership. Washington: NQF, 2018. Available: http://www.qualityforum.org/map/ [Accessed 16 Oct 2019].

6 Van Spall HGC, Rahman T, Mytton O, et al. Comparative effectiveness of transitional care services in patients discharged from the hospital with heart failure: a systematic review and network metaanalysis. Eur J Heart Fail 2017;19:1427-43.
7 Health Quality Ontario. Adopting a common approach to transitional care planning: helping health links improve transitions and coordination of care. Toronto: health quality Ontario, 2014. Available: http://www.hqontario.ca/Portals/0/documents/qi/health-links/bpimprove-package-traditional-care-planning-en.pdf [Accessed 26 Sep 2018].

8 National Quality Forum. All-Cause admissions and readmissions measures. Washington: NQF, 2015. Available: https://www. qualityforum.org/Publications/2015/04/All-Cause_Admissions_and_ Readmissions_Measures_-_Final_Report.aspx [Accessed 16 Oct 2019].

9 NTOCC Measures Work Group. Transitions of care measures. National transitions of care coalition, 2008. Available: http://www. ntocc.org/portals/0/pdf/resources/transitionsofcare_measures.pdf [Accessed 26 Sep 2018].

10 Mainz J. Defining and classifying clinical indicators for quality improvement. Int J Qual Health Care 2003;15:523-30.

11 Cochrane Effective Practice and Organisation of Care Review Group. Data collection checklist: cochrane effective practice and organisation of care, 2002. Available: https://epoc.cochrane.org/ sites/epoc.cochrane.org/files/uploads/datacollectionchecklist.pdf [Accessed 26 September 2018].

12 National Institutes of Health. Quality assessment tool for before-after (pre-post) studies with no control group, 2014. Available: https:// www.nhlbi.nih.gov/health-topics/study-quality-assessment-tools [Accessed 26 September 2018].

13 Higgins JPTet al. Measuring inconsistency in meta-analyses. BMJ 2003;327:557-60. 